ESAIM: PROCEEDINGS, March 2012, Vol. 35, p. 239-244

Fédération Denis Poisson (Orléans-Tours) et E. Trélat (UPMC), Editors

\title{
A HYBRID CLASSICAL-QUANTUM APPROACH FOR ULTRA-SCALED CONFINED NANOSTRUCTURES : MODELING AND SIMULATION*
}

\author{
Naoufel Ben Abdallah ${ }^{1}$, Clément Jourdana ${ }^{1,2}$, Paola Pietra ${ }^{2}$ and Nicolas \\ VAUCHELET $^{3}$
}

\begin{abstract}
We propose a hybrid classical-quantum model to study the motion of electrons in ultrascaled confined nanostructures. The transport of charged particles, considered as one dimensional, is described by a quantum effective mass model in the active zone coupled directly to a drift-diffusion problem in the rest of the device. We explain how this hybrid model takes into account the peculiarities due to the strong confinement and we present numerical simulations for a simplified carbon nanotube.

Résumé. Nous proposons un modèle hybride classique-quantique pour décrire le mouvement des électrons dans des nanostructures très fortement confinées. Le transport des particules, consideré unidimensionel, est décrit par un modèle quantique avec masse effective dans la zone active couplé à un problème de dérive-diffusion dans le reste du domaine. Nous expliquons comment ce modèle hybride prend en compte les spécificités de ce très fort confinement et nous présentons des résultats numériques pour un nanotube de carbone simplifié.
\end{abstract}

\section{INTRODUCTION}

The extreme miniaturization reached by the electronic devices brings the necessity of using new models to describe the electron transport, in order to predict their behavior, to access their performance limits and to design new configurations. In ultra-scaled strongly confined structures (like nanowires and nanotubes), the dimension of the transversal cross section is so thin that the transport of charged particles is confined in the one dimensional (1D) longitudinal direction. When the cross-section diameter is below $3 \mathrm{~nm}$, the strong confinement affects the energy band structure and bulk material quantities cannot be used in the simulations. In particular, the assumption of infinite periodic structure in the wire cross-section, which allows to derive the usual effective mass theorem, is not reasonable anymore.

In [4], a quantum effective mass model has been derived by performing an asymptotic process which consists in using an envelope function decomposition to obtain a new effective mass approximation (see [1] for 3D periodic crystals). We consider an infinite wire defined in a physical domain $\mathbb{R} \times \omega_{\epsilon}$, where $\epsilon$ is the typical spacing between lattice sites. As starting point, the transport is described by a scaled Schrödinger equation in $\mathbb{R} \times \omega_{\epsilon}$ containing a potential $W_{\mathcal{L}}$ generated by the crystal lattice, fast oscillating in the scale defined by the

\footnotetext{
* This work has been partially supported by the Galilée project no 25992ND of the Hubert Curien program of the Egide association and by the ANR project QUATRAIN

${ }^{1}$ Institut de Mathématiques de Toulouse, Université Paul Sabatier, 118 Route de Narbonne, 31062 Toulouse, France;

${ }^{2}$ Istituto di Matematica Applicata e Tecnologie Informatiche - CNR, Via Ferrata 1, 27100 Pavia, Italy;

e-mail: clement@imati.cnr.it \& pietra@imati.cnr.it

${ }^{3}$ UPMC Univ Paris 06, UMR 7598, Laboratoire Jacques-Louis Lions, F-75005, Paris, France; e-mail: vauchelet@ann.jussieu.fr
}

(C) EDP Sciences, SMAI 2012 
crystal spacing, and a slowly varying potential $V$ computed self-consistently through the resolution of a Poisson equation in the whole domain. Since the $2 \mathrm{D}$ cross-section $\omega_{\epsilon}$ comprises few ions, $W_{\mathcal{L}}$ is considered periodic only in the longitudinal $x$-direction, also called transport direction. The variable $z$ of the transverse section can be considered as fast variable, and it can be rescaled as $z^{\prime}=\frac{z}{\epsilon}$. $\omega$ denotes the scaled cross-section. We assign to our structure the following Bloch problem in the $3 \mathrm{D}$ cell $\mathcal{U}=(-\epsilon / 2, \epsilon / 2) \times \omega$ :

$$
\left\{\begin{array}{c}
-\frac{\hbar^{2}}{2 m_{e}} \Delta \chi_{n}+W_{\mathcal{L}} \chi_{n}=E_{n} \chi_{n} \\
\chi_{n}\left(y, z^{\prime}\right)=0 \text { on } \partial \omega_{z^{\prime}}, \quad \chi_{n} \quad \epsilon \text {-periodic in } y \\
\int_{\mathcal{U}}\left|\chi_{n}\right|^{2} d y d z^{\prime}=1
\end{array}\right.
$$

where $\hbar$ is the reduced Planck constant, $m_{e}$ is the electron mass and $y$ denotes the transport variable in the cell. This definition of the Bloch problem is peculiar to the strongly confined structure. First, the cell $\mathcal{U}$ comprises the entire cross-section such that the Bloch functions depend on the device under consideration. Second, the boundary conditions are representative of our problem since we consider the periodicity only in the transport direction and we choose homogeneous Dirichlet conditions in the other directions in order to impose confinement.

The asymptotic process [4] brings into play some relevant averaged quantities, based on the Bloch functions, which are incorporated into the quantum electron transport modeled by an infinite set of Schrödinger equations, one for each band $n$. In particular, a $n^{t h}$ band effective mass $m_{n}^{*}$ is defined by

$$
\frac{m_{e}}{m_{n}^{*}}=1-\frac{2 \hbar^{2}}{m_{e}} \sum_{n^{\prime} \neq n} \frac{P_{n n^{\prime}} P_{n^{\prime} n}}{E_{n}-E_{n^{\prime}}}, \quad \text { where } \quad P_{n n^{\prime}}=\int_{\mathcal{U}} \partial_{y} \chi_{n^{\prime}}\left(y, z^{\prime}\right) \chi_{n}\left(y, z^{\prime}\right) d y d z^{\prime} .
$$

Also, an effective potential is given by

$$
V_{n n}(x)=\int_{\omega} V\left(x, \epsilon z^{\prime}\right) g_{n n}\left(z^{\prime}\right) d z^{\prime}, \quad \text { with } \quad g_{n n}\left(z^{\prime}\right)=\int_{-\epsilon / 2}^{\epsilon / 2}\left|\chi_{n}\left(y, z^{\prime}\right)\right|^{2} d y .
$$

The quantities $g_{n n}$ contains the atomistic information of the strongly confined cross-section. They allow to make the link between the one dimensional transport direction and the three dimensional nanostructure.

In [7], a non-ballistic transport is considered where the evolution of charged particles is mainly driven by collisions with phonons. The previous effective quantities are inserted in a novel drift-diffusion equation. Here, we propose a hybrid classical-quantum approach. In a bounded domain $\left(x_{L}, x_{R}\right) \times \omega_{\epsilon}$ such that $\left|x_{R}-x_{L}\right|>>\epsilon$, we spatially couple the transport equations developed respectively in $[4,7]$. This hybrid model is mainly motivated by two reasons. Quantum effects generally take place in a localized region. Moreover, our quantum model does not include collisions of charged particles. We study here a gate all-around Field Effect Transistor (FET). It contains an active zone sandwiched between two largely doped regions (Source and Drain) considered as electron reservoirs. Thus, we use the quantum effective mass model [4] in the active zone (where quantum effects are strong) and couple it to the drift-diffusion one [7] in the rest of the domain (since collisions with phonons play an important role in reservoirs). Following the idea of [2], the coupling is made directly, getting an analytic expression of the interface conditions (ICs) by writing the exact continuity of the current. In the following, we describe this hybrid approach and we present numerical simulations for a simplified one wall carbon nanotube, emphasizing the computational efficiency of this approach.

\section{Presentation of the hybrid strategy}

We present here the hybrid strategy to couple, spatially in the transport direction, the Schrödinger system with the drift-diffusion one. We assume that the transport domain $\left(x_{L}, x_{R}\right)$ is decomposed into a quantum zone $\omega_{Q}=\left(x_{1}, x_{2}\right)$, with $x_{L}<x_{1}<x_{2}<x_{R}$ and a classical zone $\omega_{C}=\left(x_{L}, x_{R}\right) \backslash \omega_{Q}$. At this point, we assume that the electrostatic potential $\mathrm{V}$ is given (and consequently also the effective potentials $V_{n n}$ ). 


\subsection{The classical regions}

We consider the following stationary drift-diffusion model [7] on the disconnected domain $\omega_{C}$

$$
\frac{d}{d x} J_{C}(x)=0, \quad \text { with } \quad J_{C}(x)=-\mu(x) \partial_{x} \varphi(x) F\left(V_{s}(x)-\varphi(x)\right),
$$

with Dirichlet boundary conditions (imposed under the hypothesis of quasi-neutrality). $\varphi$ is the unknown quasi-fermi energy, $\mu$ is an electron mobility coefficient and $V_{s}$ is an effective potential defined by

$$
V_{s}(x)=-k_{B} T \ln \mathcal{Z}(x) \quad \text { with } \quad \mathcal{Z}(x)=\sum_{n=1}^{+\infty} e^{-\left(E_{n}+V_{n n}(x)\right) /\left(k_{B} T\right)}
$$

where $k_{B}$ is the Boltzmann constant and $T$ is the lattice temperature. In this work, we consider the Boltzmann statistics, so that $F$ is expressed by $F(s)=n_{i} e^{-s /\left(k_{B} T\right)}$, where $n_{i}$ is the intrinsic density. In view of selfconsistent computations, we also define, from the surface density $F\left(V_{s}-\varphi\right)$, a charge density for each $n^{\text {th }}$ band (see [7] for details)

$$
N_{C}^{n}(x)=F\left(V_{s}(x)-\varphi(x)\right) \frac{e^{-\left(E_{n}+V_{n n}(x)\right) /\left(k_{B} T\right)}}{\mathcal{Z}(x)}, \quad x \in \omega_{C} .
$$

Finally, the two classical regions are connected by the conditions $J_{C}\left(x_{1}\right)=J_{C}\left(x_{2}\right)=h\left(\varphi\left(x_{1}\right)\right)-h\left(\varphi\left(x_{2}\right)\right)$, with $h$ a real-valued and monotonously increasing function which will be determined by the quantum current expression.

\subsection{The quantum region}

For each $n^{\text {th }}$ band and for each wave vector $k$, we consider the following scattering states Schrödinger equation [4] on the quantum domain $x \in \omega_{Q}$

$$
-\frac{\hbar^{2}}{2 m_{n}^{*}} \partial_{x x} \psi_{n}^{k}(x)+V_{n n}(x) \psi_{n}^{k}(x)=\mathcal{E}_{n, k} \psi_{n}^{k}(x), \quad \text { with } \quad \mathcal{E}_{n, k}= \begin{cases}\mathcal{E}_{n, k}^{1}=\frac{\hbar^{2} k^{2}}{2 m_{n}^{*}}+V_{n n}\left(x_{1}\right) & \text { if } k>0 \\ \mathcal{E}_{n, k}^{2}=\frac{\hbar^{2} k^{2}}{2 m_{n}^{*}}+V_{n n}\left(x_{2}\right) & \text { if } k<0 .\end{cases}
$$

Transparent Boundary Conditions (TBCs) $[3,4]$ are used to complete the system. Next, the 1D density carried by the $n^{t h}$ band is given superimposing the densities of states injected from Source and Drain, that is

$$
N_{Q}^{n}(x)=\int_{\mathbb{R}} \phi_{n}(k)\left|\psi_{n}^{k}(x)\right|^{2} d k, \quad x \in \omega_{Q},
$$

where $\phi_{n}(k)$ is a given distribution function, expressed in term of the quasi-fermi energy variable at boundaries

$$
\phi_{n}(k)= \begin{cases}f_{n}\left(E_{n}+\mathcal{E}_{n, k}^{1}-\varphi\left(x_{1}\right)\right) & \text { if } k>0, \\ f_{n}\left(E_{n}+\mathcal{E}_{n, k}^{2}-\varphi\left(x_{2}\right)\right) & \text { if } k<0 .\end{cases}
$$

In the case of the Boltzmann statistics that we consider here, $f_{n}$ is defined by $f_{n}(s)=\frac{n_{i} \hbar}{\sqrt{2 \pi k_{B} T m_{n}^{*}}} e^{-s /\left(k_{B} T\right)}$. Finally, we can prove that the current density is constant on $\omega_{Q}$ and it is defined by

$$
J_{Q}=\sum_{n=1}^{+\infty} J_{Q}^{n}, \quad \text { with } \quad J_{Q}^{n}=\frac{q \hbar}{m_{n}^{*}} \int_{0}^{+\infty} k T_{n}(k) f\left(E_{n}+\mathcal{E}_{n, k}^{1}\right)\left(e^{\varphi\left(x_{1}\right) /\left(k_{B} T\right)}-e^{\varphi\left(x_{2}\right) /\left(k_{B} T\right)}\right) d k,
$$

where $J_{Q}^{n}$ is the $n^{t h}$ band current density and $T_{n}(k)$ the transmission coefficients. The term containing $\varphi$ enters as a multiplication factor. This is crucial to write the ICs in an explicit way, as we shall see in the next section. 


\subsection{The interface conditions}

Following the idea of [2], we build the ICs by preserving the continuity of the total current between the classical and the quantum domains. For the Boltzmann statistics, we immediately see that the quantum current can be written as the difference of an increasing function $h$ of the quasi-fermi energy variable $\varphi$ at the boundaries $x_{1}$ and $x_{2}$, as announced in Section 1.1. Then, the explicit expression of $h$ is given by

$$
h(\varphi)=\Theta^{-1} e^{\varphi /\left(k_{B} T\right)}, \quad \text { where } \quad \Theta^{-1}=\sum_{n=1}^{+\infty} \frac{q \hbar}{m_{n}^{*}} \int_{0}^{+\infty} f\left(E_{n}+\mathcal{E}_{n, k}^{1}\right) k T_{n}(k) d k
$$

is a positive number. Consequently, we obtain the final ICs

$$
J_{C}\left(x_{1}\right)=J_{C}\left(x_{2}\right)=: J_{C} \quad \text { and } \quad e^{\varphi\left(x_{1}\right) /\left(k_{B} T\right)}-e^{\varphi\left(x_{2}\right) /\left(k_{B} T\right)}=\Theta J_{C} .
$$

\subsection{The self-consistent computations}

The charge density of the hybrid model in the $n^{t h}$ band $N_{1 D}^{n}$ is defined by $N_{1 D}^{n}(x)=N_{Q}^{n}(x)$ for $x \in \omega_{Q}$ and $N_{1 D}^{n}(x)=N_{C}^{n}(x)$ for $x \in \omega_{C}$. As explained in [4,7], the transformation from the one dimensional transport direction to the entire nanowire is done by the quantities $g_{n n}(3)$. It leads to

$$
\rho(x, z)=\sum_{n=1}^{+\infty} N_{1 D}^{n}(x) g_{n n}\left(\frac{z}{\epsilon}\right) \quad(x, z) \in\left(x_{L}, x_{R}\right) \times \omega_{\epsilon} .
$$

The peculiarity comes in the combination of the confinement information of the cross-section with the one dimensional transport density. To take into account many particle effects, we consider self-consistent computations. So, we couple the transport equations with the following Poisson equation for the electrostatic potential $V_{P}$

$$
-\nabla\left(\epsilon_{r}(z) \nabla V_{P}(x, z)\right)=\frac{q}{\epsilon_{0}}\left(N_{D}(x, z)-\rho(x, z)\right), \quad(x, z) \in\left(x_{L}, x_{R}\right) \times \omega_{\epsilon},
$$

where $\epsilon_{0}$ is the permittivity in vacuum, $\epsilon_{r}$ the relative permittivity and $N_{D}$ the prescribed doping density. Then, $V$ in (3) is given by $V=-q V_{P}$.

\section{Numerical Simulations}

\subsection{Modeled device}

To test the capability of the model to describe the electron transport in an ultra-scaled confined structure, we study a very simplified case (see Fig.1), that is a one-wall carbon nanotube with a cross-section made of 12 atoms disposed on a squared frame, surrounded by one atom layer of dielectric. The transport problem is solved for a gate-all-around FET with channel length equal to $10 \mathrm{~nm}$ doped with a donor concentration equal to $N_{D}^{-}=10^{21} \mathrm{~m}^{-3}$, with Source and Drain regions $10 \mathrm{~nm}$ long, largely doped $\left(N_{D}^{+}=10^{26} \mathrm{~m}^{-3}\right)$. The cross-section edge $(\approx 2 \mathrm{~nm})$ is tiny compared to the longitudinal length equals to $30 \mathrm{~nm}$.

\subsection{Algorithm}

The first step is the resolution of the Bloch problem (1). It provides $E_{n}, m_{n}^{*}(2)$ and $g_{n n}(3)$ for each $n^{\text {th }}$ band and it is computed only once for a given device. The potential $W_{\mathcal{L}}$ is chosen as in [8]. The second step is the resolution of the coupled Schrödinger drift-diffusion Poisson system, where the physical quantities computed in the previous step are included. First, we consider the whole system at thermal equilibrium (for zero applied Drain-Source voltage $V_{D S}$ ). Next, we solve it when a $V_{D S}$ is applied, incrementing the voltage by steps of 0.02 V. Finally, we repeat the procedure for different gate voltage $V_{G}$. 

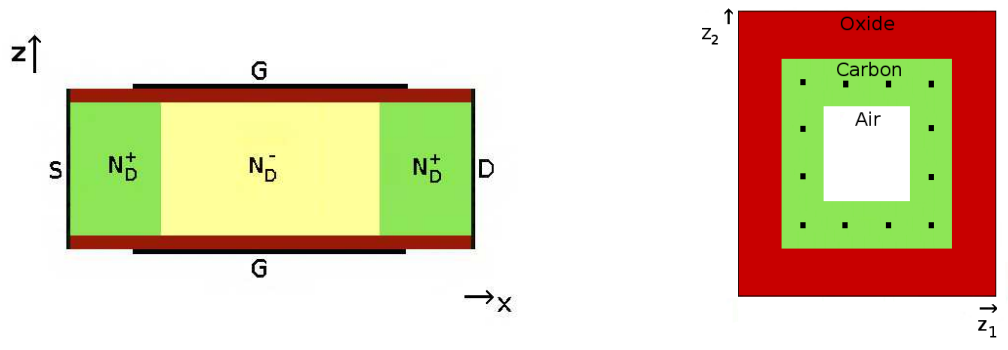

Figure 1. Longitudinal (left) and transversal (right) sections of the simplified carbon nanotube.

To treat the strong nonlinearity between the density and the potential equations, we use a Gummel iterative method $[5,6]$. At each Gummel iteration, we make the following steps. For a given $V$, we compute the potentials $V_{n n}$ (3). Next, we solve the 1D Schrödinger equations for each $n$ and $k(6)$ on $\omega_{Q}$. We obtain the wave functions $\psi_{n}^{k}$ and thus the transmission coefficients $T_{n}(k)$. It allows to compute $\Theta(9)$. Solving, by means of mixed finite elements, the equation (4) on $\omega_{C}$ with the ICs (10), we obtain $\varphi$ and thus the classical densities $N_{C}^{n}(5)$. Moreover, $\varphi$ in $x_{1}$ and $x_{2}$ allows to determine the quantum densities $N_{Q}^{n}(7)$. Finally, we compute $\rho(11)$ and the 3D Poisson equation (12), modified according to the Gummel algorithm, gives the new potential $V$.

\subsection{Numerical results}

In order to compare the hybrid approach with the quantum one, we run our Fortran90 code on the same machine and for the same device data than in [4]. The only difference are the blocks relative to the transport strategy. The hybrid method is computationally more efficient in the sense that, to reach convergence at equilibrium, it needs 6 Gummel iterations (instead of 12 for the quantum one). Also, in average, a single iteration is faster since the resolution of the large number of Schrödinger equations (obviously more expensive than the resolution of the single drift-diffusion equation) is done on a shorter domain.

Moreover, the hybrid approach allows to perform computation for large $V_{G}$, while the Gummel method breaks for $V_{G} \geq 0$ with the quantum model, due to the lack of quasi-neutrality at the boundary, which induces a wrong behavior of $V$. In Fig.2, we present $V$ (left) and $\rho$ (right) integrated over the 2D cross section, for different $V_{D S}$ and for a fixed $V_{G}=-0.1 \mathrm{~V}$ (top) or $V_{G}=0.05 \mathrm{~V}$ (bottom). Variations at $x=5$ and $25 \mathrm{~nm}$ are due to the gate and variations at $x=10$ and $20 \mathrm{~nm}$ are due to the doping. The interfaces are placed at $x=10$ and $20 \mathrm{~nm}$ and we point out that there the density is discontinuous for large $V_{D S}$ (the ICs being built to preserve only the current continuity). This brings flexibility to the system and the Gummel algorithm reaches convergence.

Fig.3 (left) represents a 2D slice of $\rho$ in logarithmic scale along the transport direction. Comparing the thermal equilibrium figure (top) with the one for $V_{D S}=0.2 \mathrm{~V}$ (bottom), we observe a channel formation in correspondence to each nucleus and the electron motion from Source to Drain. In Fig.3 (right), we present the Current-Voltage curves for various $V_{G}$. As expected, the current in the FET increases with $V_{G}$. We observe the two typical regimes: an ohmic regime for small $V_{D S}$ and a quasi-saturation regime for larger $V_{D S}$. This saturation regime is established for larger $V_{D S}$ when $V_{G}$ increases. All these observations are in accordance with the behavior of conventional FETs. We conclude that our computationally efficient hybrid model is qualitatively able to capture the transport of electron in ultra-scaled confined nanostructures, even for a very simplified device.

\section{REFERENCES}

[1] L. Barletti and N. Ben Abdallah. Quantum Transport in Crystals: Effective Mass Theorem and K.P Hamiltonians. Comm. Math. Phys., 307:567-607, 2011.

[2] M. Baro, N. Ben Abdallah, P. Degond, and A. El Ayyadi. A 1d coupled Schrödinger drift-diffusion model including collisions. J. Comput. Phys., 203(1):129 - 153, 2005. 

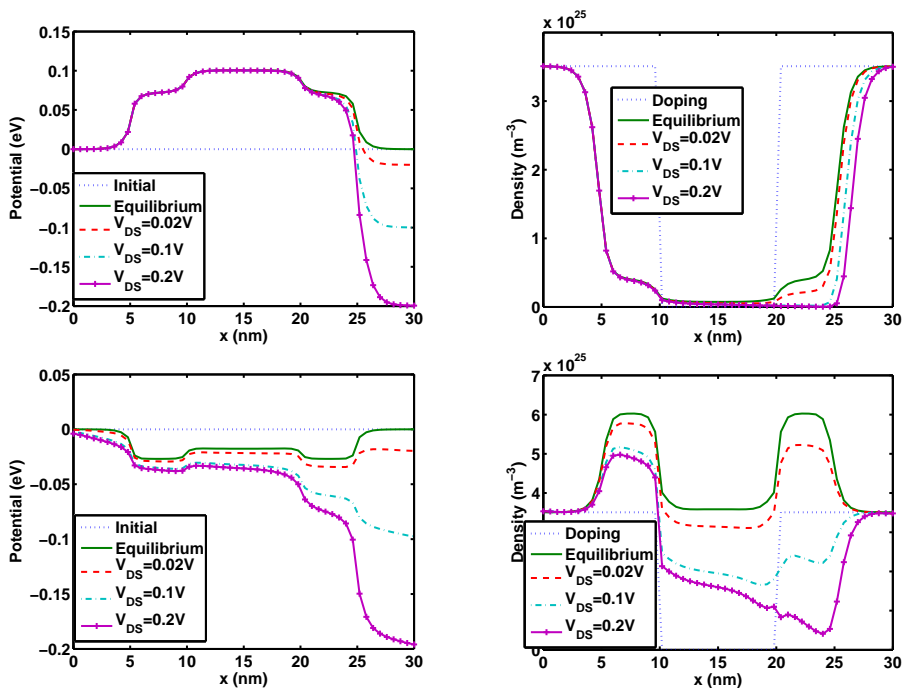

Figure 2. 1D potential energies (eV) (left) and 1D densities $\left(m^{-3}\right)$ (right) for different $V_{D S}$, with $V_{G}=-0.1 \mathrm{~V}$ (top) and $V_{G}=0.05 \mathrm{~V}$ (bottom).
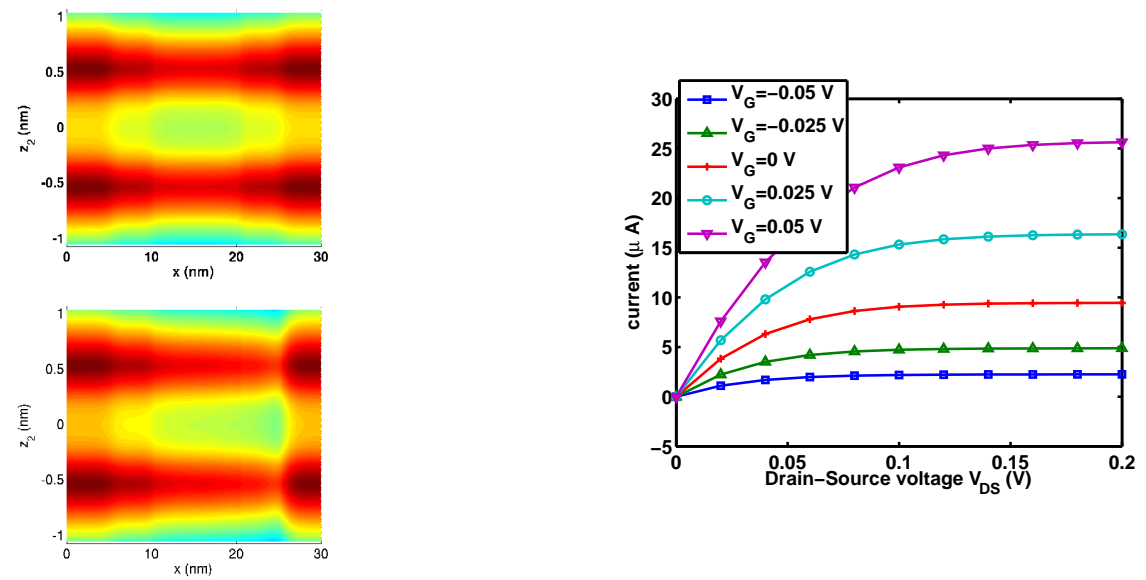

FIGURE 3. Left: 2D slice (crossing 2 nuclei) of the density in logarithmic scale at equilibrium (top) and for $V_{D S}=0.2 \mathrm{~V}$ (bottom), with $V_{G}=-0.1 \mathrm{~V}$. $x$-axis is the transport direction. Right: Current-Voltage characteristics for five gate voltages $V_{G}$.

[3] N. Ben Abdallah, P. Degond, and P. A. Markowich. On a one-dimensional Schrödinger-Poisson scattering model. Z. Angew. Math. Phys., 48(1):135-155, 1997.

[4] N. Ben Abdallah, C. Jourdana, and P. Pietra. An effective mass model for the simulation of ultra-scaled confined devices. Preprint IMATI-CNR 10PV11/7/0.

[5] Ph. Caussignac, B. Zimmermann, and R. Ferro. Finite element approximation of electrostatic potential in one-dimensional multilayer structures with quantized electronic charge. Computing, 45(3):251-264, 1990.

[6] H.K. Gummel. A self-consistent iterative scheme for one-dimensional steady state transistor calculations. IEEE Trans. Electron Devices, 11(10):455 - 465, 1964.

[7] C. Jourdana and N. Vauchelet. Analysis of a diffusive effective mass model for nanowires. Kinet. Relat. Models, 4:1121-1142, 2011.

[8] D. Zhang and E. Polizzi. Efficient modeling techniques for atomistic-based electronic density calculations. J. Comput. Electron., $7: 427-431,2008$. 